\title{
A Case of Commotio Cordis Caused by Steering Wheel Injury
}

\author{
Noriyoshi Kutsukata, Kunihiro Mashiko, Hisashi Matsumoto, \\ Yoshiaki Hara, Yuichiro Sakamoto and Hiroyuki Koami \\ Department of Emergency and Critical Care Medicine, Graduate School of Medicine, Nippon Medical School \\ Shock and Trauma Center, Nippon Medical School Chiba Hokusoh Hospital
}

\begin{abstract}
We report a rare case of commotio cordis caused by traffic injury. The patient was a 60 year-old female driver who suffered severe steering wheel impact to the chest during a headon collision in which her car overturned. She had no history of cardiac disease. Emergency medical services arrived at the scene within 12 minutes of the accident. Evidence of ventricular fibrillation led the paramedics to carry out immediate defibrillation with an automated external defibrillator. Restoration of spontaneous circulation was confirmed within 2 minutes, along with establishment of sinus rhythm and normal wave form on electrocardiography. The patient was transported to our hospital in an emergency helicopter. General examination revealed chest bruising, and computed tomography of the chest showed pulmonary contusions; there was no other evidence of critical injury. We performed endotracheal intubation, as the patient had consciousness disturbance, and then initiated hypothermic therapy in the intensive care unit. Meanwhile, the hemodynamics remained stable, and there was no recurrence of arrhythmia. On day 15, the patient's consciousness improved, and she was able to communicate. Two months later, she was transported to another hospital for rehabilitation.
\end{abstract}

(J Nippon Med Sch 2010; 77: 218-220)

Key words: commotio cordis, traffic accident, traffic injury, steering wheel injury

\section{Introduction}

Commotio cordis is a rare event, and most reports describe its occurrence during sporting activities in pediatric patients. There have been very few reports of commotio cordis caused by traffic injury, and those that exist point to a low survival rate. We report a case caused by traffic injury in a middleaged woman, who was successfully resuscitated.

\section{Case Report}

The patient was a 60-year-old woman with no history of cardiac risk factors or heart failure. She was involved in a head-on collision at a traffic crossing, and her car overturned. The front of the car was crushed, and the windshield shattered. The car was not equipped with airbags, and the patient was not wearing a seatbelt. During the accident, she hit her chest hard against the steering wheel. There

Correspondence to Noriyoshi Kutsukata, Shock and Trauma Center, Nippon Medical School Chiba Hokusoh Hospital, 1715 Kamagari, Inzai, Chiba 270-1694, Japan

E-mail: moonsong@nms.ac.jp

Journal Website (http://www.nms.ac.jp/jnms/) 


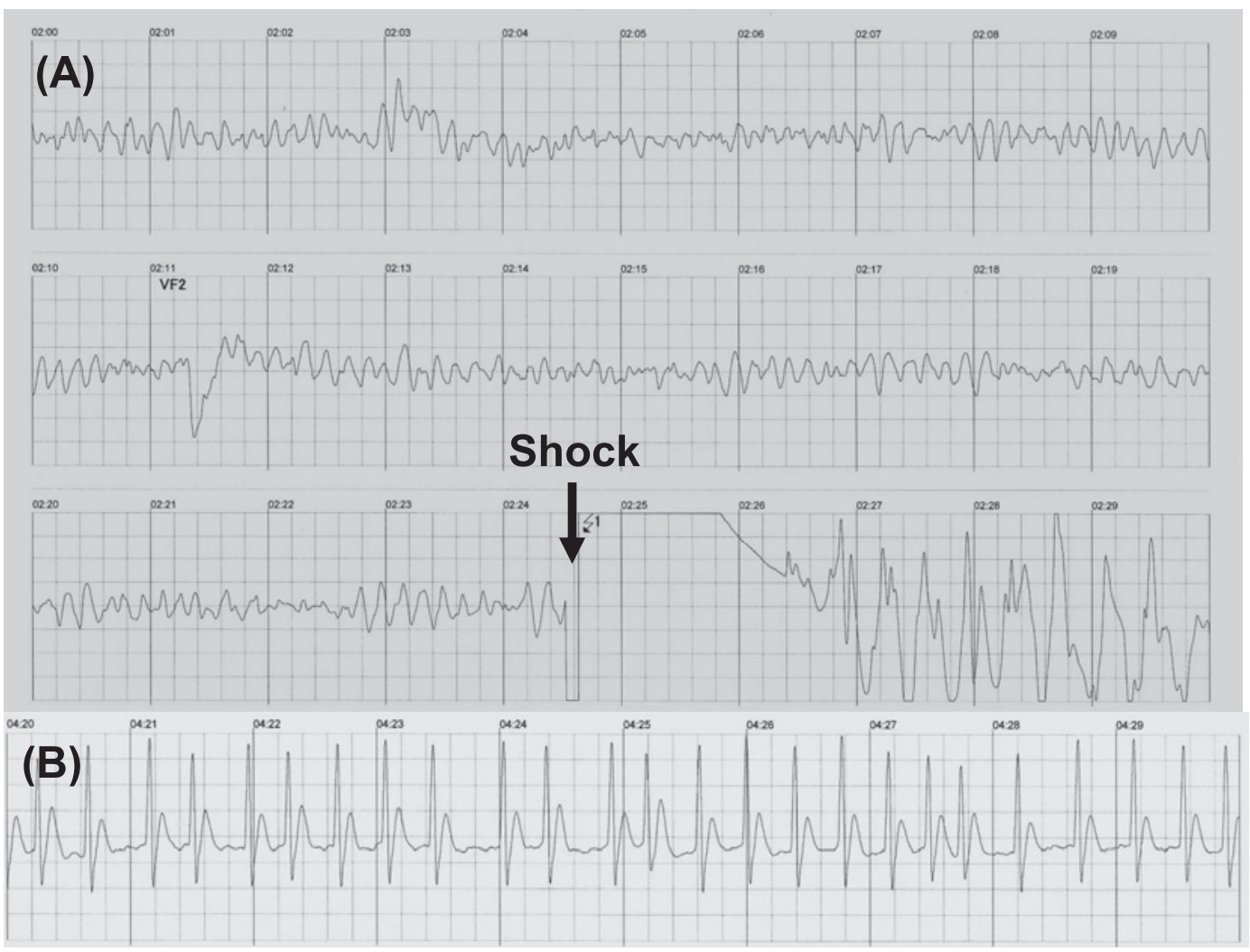

Fig. 1 Evidence of ventricular fibrillation was found, and defibrillation was carried out with an AED immediately (A). Return of spontaneous circulation (ROSC) was restored within 2 minutes of defibrillation (B).

were no bystander rescuers, but emergency medical services were on the scene within 12 minutes of the accident, and paramedics performed cardiopulmonary resuscitation (CPR), because ventricular fibrillation was detected. For the same reason, defibrillation with an automated external defibrillator (AED) was also carried out immediately. Return of spontaneous circulation (ROSC) was restored within 2 minutes of defibrillation, and establishment of normal sinus rhythm was confirmed (Fig. 1).

The patient was immediately transported to our hospital in an emergency helicopter. On arrival, she was hemodynamically stable, and sonography revealed no significant findings. General physical examination revealed a mild hematoma in the chest wall (midline) resulting from blunt trauma, and computed tomography (CT) of the chest showed pulmonary contusions. Vital signs were stable (blood pressure 106/60 $\mathrm{mm} \mathrm{Hg}$; heart rate $65 \mathrm{bpm}$; respiratory rate $23 \mathrm{bpm}$; temperature $36.3^{\circ} \mathrm{C}$ ), and chest examination revealed bilateral crackles. Serum creatine phosphokinase levels were normal, and Troponin $\mathrm{T}$ test was negative. No evidence of any other critical injury was detected. We performed endotracheal intubation, as the patient showed disturbance of consciousness (Glasgow Coma Scale score 3=E1 V1 M1, with bilateral pupillary miosis), and then initiated hypothermic therapy $\left(34^{\circ} \mathrm{C} ; 24\right.$ hours) in the intensive care unit (ICU). The patient remained in the ICU for 3 days.

During the clinical course, the patient remained hemodynamically stable, and there was no recurrence of arrhythmia. On day 15, she was able to communicate, and 2 months later, she was transferred to a nursing facility for rehabilitation.

\section{Discussion}

Commotio cordis is a rare disorder of sudden onset. When the offending impact occurs 10-30 msec before the peak of the $\mathrm{T}$ wave in the cardiac cycle, critical arrhythmias, such as ventricular fibrillation 
Table 1 Reported cases of cardiac concussion caused by traffic injury

\begin{tabular}{ccccc}
\hline Case no. & Age $(\mathrm{yr}) /$ Gender & Type of injury & Outcome & First Author (Year) \\
\hline 1 & $34 / \mathrm{M}$ & Ambulance driver & Dead & Michalodimitrakis et al. (1997) \\
2 & $21 / \mathrm{M}$ & Motorcycle rider & Dead & Michalodimitrakis et al. (1997) \\
3 & $38 / \mathrm{M}$ & Car driver & Alive & Loren et al. (1997) \\
4 & $21 / \mathrm{M}$ & Road accident & Alive & Cotter et al. (1996) \\
5 & $16 / \mathrm{M}$ & Road accident & Dead & Cotter et al. (1996) \\
6 & $23 / \mathrm{M}$ & Car driver & Alive & Cotter et al. (1996) \\
\hline
\end{tabular}

develop ${ }^{1}$, and about $84 \%$ of those affected die despite aggressive attempts at resuscitation ${ }^{2}$. According to the American cording registry, a total of 128 cases of commotio cordis occurring during sporting activities have been reported. The condition can result, for example, from the strong impact of a baseball on the precordium. In most pediatric patients, the impact characteristically involves the anterior chest wall. Reports of commotio cordis occurring as a result of traffic injury are very rare.

The following diagnostic criteria were proposed by Maron:

1. witnessed occurrence of a blunt, nonpenetrating blow to the chest immediately preceding cardiovascular collapse;

2. detailed documentation of the circumstances surrounding the blow from available newspaper articles, police reports, and telephone interviews with family members or other witnesses;

3. absence of structual damage to the sternum, ribs, or the heart itself;

4. absence of any underlying cardiovascular abnormalities.

Our patient showed mild pulmonary contusions on CT, but satisfied the above criteria. She did not receive bystander $\mathrm{CPR}$, and it was 12 minutes before the emergency team arrived and instituted $\mathrm{CPR}$, and 16 minutes before ROSC could be effected. Bystander CPR is generally considered necessary to prevent subsequent complications in such patients.

Recent developments in automotive engineering have greatly improved collision safety and reduced traffic accident injuries. Unfortunately, our patient was not wearing a seat belt, and her car did not have an airbag system installed. Moreover, the car was a compact model with an engine displacement of less than $660 \mathrm{cc}$ and a total length of less than 3.4 $\mathrm{m}$. We believe that commotio cordis in this patient was caused by direct impact to her chest.

Of the 6 reported cases of commotio caused by vehicular accidents that we found, 3 victims survived (Table 1) ${ }^{3-5}$. Immediate resuscitation with an AED appears to be key to survival. It must be recognized that commotio cordis can result from traffic injuries, and the chain of survival (early access, early CPR, early defibrillation, and early advanced care) should be carried out.

\section{References}

1. Links MS, Maron BJ, VanderBrink BA, et al.: Impact directly over the cardiac silhouette is necessary to produce ventricular fibrillation in an experimental model of commotio cordis. J Am Coll Cardiol 2001; 37: 649-654.

2. Maron BJ, Gohman TE, Kyle SB, et al.: Clinical profile and spectrum of commotion cordis. JAMA 2002; 287: 1142-1146.

3. Michalodimitrakis EN, Tsatsakis JD, Aristidis M: Vehicular accidents and cardiac concussion: a traumatic connection. Am J Forensic Med 1997; 18: 282-284.

4. Crown LA, Hawkins W: Commotio cordis: clinical implications of blunt cardiac trauma. Am Family Physician 1997; 55: 2467-2470.

5. Cotter G, Moshkovitz Y, Barash P, et al.: Ventricular fibrillation in the patient with blunt trauma: not always exsanguination. J Trauma 1996; 41: 345-347.

(Received, March 26, 2010)

(Accepted, April 20, 2010) 